\title{
Review of "Exposure to antiresorptive therapy with bisphosphonates does not induce histological changes in human alveolar jawbone"
}

\author{
Vittorio Fusco ${ }^{1}$ \\ 1 Azienda Ospedaliera SS Arrig o e Biagio e Cesare Arrigo
}

Definitions

Medication related osteonecrosis of the jaw (MRONJ)

Defined by Alberto Bedogni et al.

Bisphosphonate related osteonecrosis of the jaw (BRONJ)

Defined by Alberto Bedog ni et al.

Definition of medication-related osteonecrosis of the jaw in 2014

Defined by Salvatore L. Ruggiero et al.

Osteosclerosis of the jaw

Defined by Alberto Bedog ni

Authors are to be commended for the originality of the study as an attempt to enlarge basic knowledge about the multifactorial pathogenesis of

Bisphosphonate-Related Osteonecrosis of the Jaw (BRONJ). The goal of this study is noteworthy as the understanding of tissue changes in the jaw of individuals treated with NBP who don't have BRONJ has only been studied in animals. Therefore, the potential impact of this study is very sound. The sample size is a real strength of the study. However, the histological data presented are only descriptive and the lack of detailed quantitative data represents a limitation of the study, at present.

According to the most recent version of the American Association of Oral and Maxillofacial Surgery (AAOMS) consensus paper, ${ }^{[1]}$ diagnosis of MRONJ is purely clinical and related to the presence of exposed jaw bone or bone that can be probed through an intraoral/extraoral fistula. Patients with signs and symptoms other than exposed jaw bone or bone probing fistulas continue to remain excluded from MRONJ case definition. ${ }^{[2]}$ In an attempt to properly address potential ONJ patients presenting with minor clinical signs, radiological imaging and Computed Tomography (CT) in particular have been included in the diagnostic process of MRONJ (Italian consensus) to better 
reflect the actual bone extension of disease. Despite the fact that bone density alteration (osteosclerosis of the jaw) is always present in BRONJ patients, CT signs of MRONJ are non-specific and the identification of early histological patterns of disease might become essential to improve the accuracy of the diagnostic process. The present study could serve as a basement for future research in order to identify a histological counterpart for CT-recorded bone sclerosis.

From my perspective, the most reliable histopathological variables among the 20 evaluated in the study or their combinations could also be tested in bone biopsies of patients with initial signs of osteosclerosis/minor clinical signs of BRONJ, who were orig inally excluded from the present study. In fact, the presence of some histopathological bone alterations in the bone of such patients could predict early BRONJ, or at least define very high-risk patients. The cohorts of the present study may serve as negative controls against the early BRONJ cases. ${ }^{[3]}$

\section{References}

1. ^Salvatore L. Ruggiero, Thomas B. Dodson, John Fantasia, Reginald Goodday, Tara Aghaloo, Bhoomi Mehrotra. (2014). American Association of Oral and Maxillofacial Surgeons Position Paper on Medication-Related Osteonecrosis of the Jaw-2014 Update. Journal of Oral and Maxillofacial Surgery, vol. 72 (10), 1938-1956.

2. `Stefano Fedele, Giorgio Bedogni, Matteo Scoletta, Gianfranco Favia, Giuseppe Colella, Alessandro Agrillo. (2015). Up to a quarter of patients with osteonecrosis of the jaw associated with antiresorptive agents remain undiagnosed. British Journal of Oral and Maxillofacial Surgery, vol. 53 (1), 13-17.

3. ^ Giorgia Saia, Stella Blandamura, Giordana Bettini, Anita Tronchet, Andrea Totola, Giorgio Bedogni. (2010). Occurrence of Bisphosphonate-Related Osteonecrosis of the Law After Surgical Tooth Extraction. Journal of Oral and Maxillofacial Surgery, vol. 68 (4), 797-804. 